\title{
La innovación como clave para que la industria ornamental trascienda en el estado de Veracruz
}

\author{
Hernández Marañón, Felipe \\ Instituto Tecnologico de Orizaba (Egresado), México \\ felipe120893@hotmail.com
}

Resumen - En la actualidad la industria ornamental en Veracruz atraviesa un proceso de transición, de empresas familiares a negocios inteligentes para enfrentar el mercado nacional. El objetivo del presente trabajo es determinar los aspectos que se requieren para poder implementar una microempresa ornamental, la metodología consta de ocho fases, hechas para emprendedores. Los resultados del trabajo son el establecimiento de una microempresa productora y comercializadora de plantas dracenas miniatura, la cual contribuirá a satisfacer la demanda existente en el mercado ornamental.

Palabras clave: Plantas miniatura; Producción; Comercialización; Plan de negocios;

Abstract - At present, the ornamental industry in Veracruz is going through a transition process, from family businesses to smart businesses to face the national market. The objective of this work is to determine the aspects that are required to implement an ornamental microenterprise, the constant eight-phase methodology, made for entrepreneurs. The results of the work are the establishment of a microenterprise producer and marketer of miniature dragon plants, which contributes to meet the demand in the ornamental market.

Keywords: Miniature plants; Production; Marketing; Business Plan;

\section{INTRODUCCIÓN}

La producción mundial de planta y flor se ha extendido en los últimos años, con numerosos centros productivos localizados en países en desarrollo, que abastecen de forma regular a los grandes consumidores. En general, el comercio internacional ornamental sigue un eje Sur-Norte definido, con pocas conexiones transversales. Por ejemplo, Colombia y Ecuador tienen su principal mercado en los Estados Unidos de América, Kenia en Europa y los países del sudeste de Asia en Japón (Yanai., 2012).
Interconectando Saberes, 2020

ISSN: 2448=8704
Fecha de Recepción: 30 de septiembre de 2019

Fecha de Aceptación: 18 de febrero de 2020

Fecha de Publicación: 09 de marzo de 2020 
Los principales países productores de plantas ornamentales, medidos en superficie productiva actualmente son: China (con 40, 000 ha en flor cortada y 60,000 ha en planta en maceta) y la India (con 100, 000 ha tanto de flor como de planta). En cuanto al valor de la producción, los principales países son Italia, Países Bajos, Japón y Estados Unidos de Norteamérica. La producción europea continúa siendo la primera del mundo en valor, con 10, 228 millones de euros que representa el $42 \%$ de la producción mundial. Por otra parte, cabe destacar que Colombia, Ecuador y Kenia, se caracterizan porque sus mercados se orientan casi exclusivamente a la exportación (AIPH, 2014).

En México, la producción de plantas ornamentales reviste una gran importancia cultural, ambiental, social y económica a pesar de no ser un producto básico, tal como lo son las frutas, hortalizas y oleaginosas. En cuanto a las plantas ornamentales, en México se aprovechan más de 1,000 especies y variedades, ocupando una super- ficie de alrededor de 20, 000 ha, sólo el $0.1 \%$ de la superficie del país, distribuidas en 20 estados de la república y generando de 8 a 12 empleos por hectárea permanentes beneficiando alrededor de $150 \mathrm{mil}$ familias, en el año 2013 generó casi $6 \mathrm{mil}$ MDP, el $1.5 \%$ del valor del sector agrícola nacional (Secretaría de Agricultura, 2014).

Actualmente la actividad ornamental no es tan importante en el estado de Veracruz con respecto a otros estados, porque en el país son 26 entidades las que participan en la producción ornamental, de estas 26 entidades las principales son, el Estado de México con un $53 \%$ del $100 \%$ nacional, Distrito Federal $17 \%$, Jalisco, Morelos con un $8 \%$ y puebla el 6\% (Rivera, 2015). Se puede observar que Veracruz no figura en los primeros lugares a pesar de que se tienen condiciones idóneas para producir especies exóticas de alto valor en el mercado, ya que se estima que se tienen más de 1,500 hectáreas y aproximadamente 2,000 productores en el estado dedicados a la actividad ornamental. 
El mundo de las plantas ornamentales es muy extenso por lo cual existen un sin fin de familias, la familia que se abordara en este trabajo es el género "Dracena o Dracaena", este pertenece a la familia de las liliáceas y su nombre deriva de la palabra griega drakania, que significa dragona. Las plantas dracenas engloban unas 40 especies tropicales, originarias de África y Asia, que en general presentan aspecto de palmera, aunque no están emparentadas con estas últimas (Infoagro Systems, 2012).

Las plantas dracenas tipo miniatura, surge en el continente asiático debido a la necesidad de pequeños espacios verdes en grandes urbes, al llegar a México han tenido una gran aceptación, lo cual provoco la regionalización (adaptación del diseño a los productos locales) de estos productos en similares presentaciones. La mayor demanda de este tipo de plantas se centra en los principales mercados del país, en la ciudad de México existen cinco mercados con alto potencial de adquisición ubicados en la delegación Xochimilco (Xochimilco, 2008).
Otros estados como lo son Puebla y Morelos de igual manera registran demanda de plantas dracenas miniatura, ya que estos dos estados cuentan con mercados en potencial crecimiento, tales como el Municipio de Atlixco de las Flores en Puebla y en el estado de Morelos se encuentran mercados en los municipios de Cuautla, Cuernavaca y Cocoyol por citar algunos (Aldana, 2010).

Las empresas que se dedican a producir y comercializar plantas ornamentales tienen ciertos problemas, entre los cuales sobresalen la falta de organización, la incipiente infraestructura, la escasa disponibilidad de consultores que proporcionen asistencia técnica, la escasa aplicación de tecnologías y el alto número de intermediarios, por mencionar algunas de ellas (Horticultivos, 2011).

Otra característica notable es la falta de innovación en sus sistemas de producción, lo cual genera elevados costos en el proceso de estas empresas, esto afectan el precio final de sus productos, lo que ocasiona que estas entidades 
no logren ser competitivas respecto a las de otros estados, por lo cual no logran vender lo que producen y esto conlleva al poco crecimiento de estas entidades, en casos críticos el cierre de dichas (ChalateMolina, 2008).

En ámbito nacional la producción de plantas dracenas miniatura es un sector poco explotado por los productores de plantas ornamentales, ya que la mayoría de los productores se dedican a producir plantas en tamaños pequeños, medianos y así sucesivamente (Tecnoagro, 21). Pero la poca explotación del sector antes mencionado se debe a que la producción de plantas dracenas miniaturas, requiere de controles tecnológicos (hídrico) altamente especifico, debido al tamaño de las plantas son altamente susceptibles a los cambios climáticos que las pudieran afectar generando plagas y enfermedades letales en cuestión horas, (mayor control de riesgos) cada planta en su estado natural genera un microclima que la ayuda a regular cualquier tipo de estrés que se le pudiese generar, pero al ser estas unidades de producción de tamaños miniaturas requieren, de un control de humedad y a su vez recurso hídrico de manera precisa que solo se puede lograr a través de tecnologías de sistema de riego como nebulizaciones, sistemas de goteo entre otros que muchos de los productores en su mayoría desconocen (Bouchet E.R., 2007).

\section{MARCO TEÓRICO}

Un plan de negocios es un instrumento que se utiliza para documentar el propósito y los proyectos del autor, respecto a cada aspecto del negocio. El documento puede ser utilizado para comunicar los planes, estrategias y tácticas a sus administradores, socios e inversionistas. También se emplea cuando se solicitan créditos empresariales o bancarios. El plan de negocios contiene tanto objetivos estratégicos como tácticos, y puede ser informal o formal. Asimismo, puede estar en una servilleta o en su cabeza, o puede simplemente ser una lista de tareas pendientes. Si se les pregunta a los propietarios de negocios o empresarios promedio si tienen un "plan", todos dirían "por supuesto". Pídales que se lo expliquen y usted acabará muy probablemente con una perspectiva general de sus metas principales (Balanko-Dickson, 2008). 
Sin embargo, el plan de negocios no es un documento estático; por el contrario, se trata de un plan dinámico, cambiante, adaptable, que forzosamente se renueva con el tiempo. En pocas palabras, es un documento "vivo" que, de acuerdo con la respuesta y los resultados que se vayan obteniendo con el mismo, se renueva y permite reorganizar los recursos de la empresa para definir nuevas alternativas de acción que faciliten al emprendedor acercarse más a su meta. Aunque es de vital importancia iniciar el proyecto de empresa con un plan muy bien redactado, éste debe revisarse con regularidad y corregirse en función del entorno y de los éxitos que se presenten, pero también de las deficiencias. De esta forma, se configura un nuevo plan de negocios en cada momento clave del desarrollo de la empresa (Filion, Cisneros, \& Mejía-Morelos, 2011).

Un plan de negocios describe una serie de puntos esenciales para un proyecto de éxito, tales puntos pueden ser: el producto o servicio, la competencia, el mercado, la elaboración del producto y/o la prestación del servicio, el siste- ma de administración (organización), el estado financiero del proyecto, la planeación estratégica y operativa, los requisitos legales. Muchos autores nos mencionan que un plan de negocios cuenta con una serie de pasos, pero en concreto el autor (Rodríguez, 2011, pág. xvi), nos dice que un plan de negocios abarca siete grandes áreas de suma importancia (naturaleza del proyecto, el mercado del producto o servicio, sistema de producción, la organización y el recurso humano en el proyecto, el aspecto legal en que el proyecto está inmerso, las finanzas del proyecto, el proceso de planeación para el arranque, desarrollo y consolidación del proyecto (Rodríguez, 2011), estas áreas mencionadas se aprecian en los siguientes puntos:

1. Naturaleza del proyecto. Antes de iniciar cualquier negocio, es necesario definir la esencia de este, es decir cuáles son los objetivos, cuál es la misión que persigue y por qué se considera justificable su desarrollo. La naturaleza del proyecto según (Rodríguez, 2011), nos dice que está estructurada en ocho secciones o pasos, mediante los cuales vamos a definir qué es eso que llamamos negocio, dichos pasos son los siguientes: 
I. Proceso creativo para determinar el producto o servicio de la empresa. El primer elemento que se considerara en la formación de una empresa de éxito radica en lo creativo de la idea que le da origen. Las oportunidades están en cualquier parte, sólo hay que saber buscarlas.

\section{Justificación de la empre-}

sa. Una vez que ha seleccionado una idea, debe justificar la importancia de esta, es decir especificar la necesidad o carencia que satisface, o bien el problema concreto que resuelve.

\section{Propuesta de valor. La} propuesta o propuestas de valor, es aquella que tiene la fuerza para impulsar el desarrollo del emprendimiento, la idea que diferenciará un producto de elementos similares a los de la competencia.

IV. Nombre de la empresa. EI nombre de la empresa es su carta de presentación, es el reflejo de su imagen, su sello distintivo y por ende debe reunir una serie de características específicas.

\section{Descripción, localización} y tamaño de la empresa. El giro de una empresa es su objeto u ocupación principal. En México existen cuatro giros en los que toda empresa se puede clasificar, de acuerdo con la Secretaría de Economía estos giros son: comercial, industrial, servicios y social. La ubicación de la empresa permite determinar el medio ambiente cercano a ésta. El tamaño de la empresa se determina de acuerdo con la clasificación establecida por organismos de gobierno, en México se clasifican en cuatro tamaños, estos tamaños son: micro, pequeña, mediana y grande empresa.

VI. Análisis FODA. El análisis FODA es una herramienta que el emprendedor puede utilizar para valorar la viabilidad actual y futura de un proyecto, es decir es un diagnóstico que facilita la toma de decisiones.

VII. Misión. La misión de una empresa es su razón de ser; es el propósito o motivo por el cual existe y por tanto da sentido y guía sus actividades. 
VIII. Visión. Al igual que la misión, la visión de la empresa es una declaración que ayuda el emprendedor a seguir el rumbo al que se dirige a largo plazo. Una visión clara permite establecer objetivos y estrategias que se convierten en acciones que inspiren a todos los miembros del equipo para llegar a la meta.

2. El mercado. El mercado para una empresa es aquello todo que tiene que ver con actividades relacionadas con el precio, promoción, distribución y venta de productos o servicios, que el consumidor requiera con el fin de crear la relación cliente empresa (Rodríguez, 2011). Para que el mercado de la futura empresa se encuentre en los mejores términos debemos realizar seis puntos, si se realizan de la forma correcta tendremos como resultado una empresa competitiva dentro del mercado que se encuentre, los seis puntos son los siguientes:

I. Investigación de mercado. El estudio de mercado es el medio para recopilar, registrar y analizar datos en relación con el mercado específico al cual la empresa ofrece sus productos.
II. Distribución y puntos de venta. Cada paso del proceso de distribución posibilita incrementar la distribución a un mayor número de clientes potenciales, sin embargo, cada paso también provoca una pérdida de control y contacto directo con el mercado.

\section{Promoción del producto.} Promover es en esencia, un acto de información, persuasión y comunicación, que incluye varios aspectos de gran importancia, como la publicidad, la promoción de ventas, las marcas e, indirectamente, las etiquetas y el empaque.

\section{Fijación y políticas de} precio. El comprador entrega algo de valor económico al vendedor, a cambio de los bienes y servicios que se le ofrecen. La cantidad de dinero que se paga por los bienes o servicios constituye su precio. La fijación del precio es de suma importancia, ya que el precio influye en la percepción que tiene el consumidor final sobre el producto o servicio.

\section{Plan de introducción al} mercado. Es la estructuración de acciones concretas a realizar en los primeros meses de desarrollo de la empresa para garantizar el éxito al entrar en el merca- 
do; el plan debe tomar en consideración la manera en que se dará a conocer la empresa (sus productos o servicios) a los clientes potenciales y cómo se posicionará en el mercado.

\section{Sistema y plan de ven-} tas. Existen diversos sistemas de ventas que abarcan desde los personales, hasta los que implican sistemas complejos de distribución. Las ventas personales son de especial importancia para el pequeño empresario, ya que la mayor parte de las actividades en la pequeña empresa se realizan en esa forma.

3. Producción. Se refiere a la creación y el procesamiento de bienes y mercancías. Producción abarca la concepción, el procesamiento y la financiación, entre otras etapas. La producción constituye uno de los procesos económicos más importantes y es el medio a través del cual el trabajo humano genera riqueza (Rodríguez, 2011). Para poder tener un buen funcionamiento en el área de producción se deben de realizar diez puntos esenciales, con la realización de estos puntos quedara estructurada el área de producción de la empresa, los puntos mencionados son los siguientes:
I. Especificaciones del producto o servicio. El diseño debe especificar en qué consiste el producto o servicio, hacer una descripción detallada del mismo e incluir dimensiones, colores, materiales y otras características que lo definen.

\section{Descripción del proceso} de producción o prestación del servicio. El proceso de producción es el conjunto de actividades que se llevan a cabo para elaborar un producto o para prestar un servicio. En él se conjuntan la maquinaria, la materia prima y los recursos humanos necesarios para realizar el proceso.

\section{Diagrama de flujo del} proceso. El diagrama de flujo del proceso de producción es una secuencia gráfica de las operaciones; sirve para detallar y analizar el proceso de producción, mediante el uso de un diagrama de flujo.

\section{Equipo e instalaciones.}

El proceso productivo permite determinar las actividades a realizar, así como el equipo, herramientas e instalaciones requeridas para llevar a cabo la elaboración de productos y/o prestación de servicios de la empresa. 
V. Materia prima. La materia prima se refiere a los elementos, partes o sustancias de las que está compuesto el producto de la empresa o a los insumos necesarios para prestar un servicio.

VI. Capacidad instalada. La capacidad instalada se refiere al nivel máximo de producción que puede llegar a tener una empresa con base en los recursos con los que cuenta, principalmente en cuanto a maquinaria, equipo e instalaciones físicas.

VII. Manejo de inventarios.

El inventario es la cantidad de materiales que se encuentran en la empresa, ya sea materia prima, producto en proceso o producto terminado.

\section{Diseño y distribución} de planta y oficinas. El diseño y distribución de planta $y$ oficinas es la forma en que se dispondrán las máquinas, herramientas y los flujos de producción, lo cual permitirá organizar el trabajo eficientemente.

\section{Mano de obra requerida.} La mano de obra engloba a las personas que forman parte del proceso productivo o prestan el servicio, que con su esfuerzo y trabajo transforman la materia prima y obtienen el producto terminado.

\section{$X$. Procedimientos de mejora} continua. Contar con procedimientos de mejora continua constituye una ventaja competitiva para la empresa pues le permite ajustar sus procesos, productos y servicios a las necesidades de sus clientes, ahorrar trabajos $y$, por ende, conduce a una mayor productividad y reducción de gastos innecesarios.

4. Organización. La organización es una parte fundamental en cualquier tipo de proyecto, pero para concebir una empresa, es de vital importancia por lo cual se deben de tener ciertas características que a continuación se muestran (Rodríguez, 2011). Para tener una correcta administración en nuestra empresa debemos de realizar siete puntos con la correcta realización de estos puntos obtendremos una organización adecuada para nuestra empresa, esto será parte fundamental ya que si se administran de forma adecuada los recursos de la empresa obtendremos una empresa competitiva. Los siete puntos mencionados son los siguientes: 
I. Estructura organizacional. El primer paso para establecer un sistema de organización en la empresa es hacer que los objetivos de la empresa y los de sus áreas funcionales concuerden y se complementen.

\section{Funciones específicas por} puesto. En el punto anterior del plan se mencionaron las funciones generales de cada puesto, por lo que el siguiente paso es diseñar el manual operativo por trabajador.

III. Captación de personal. El ciclo de personal abarca diversas actividades que permiten encontrar, seleccionar, contratar, recibir, preparar y definir la carrera del personal que formará parte de la empresa desde el momento de su nacimiento.

\section{Desarrollo del personal.} El desarrollo del personal involucra dos elementos sumamente parecidos, pero con diferentes objetivos $y$, por lo tanto, con diversas formas de diseño y ejecución, éstos son adiestramiento capacitación.

\section{Administración de suel-} dos y salarios. Cuando se cuenta con una descripción básica de desempeños, perfiles por puesto y organigra- ma de la empresa, el proceso de administrar sueldos y salarios resulta muy sencillo y rápido de realizar pues sólo implica jerarquizar los puestos de la empresa.

\section{Evaluación del desem-} peño. La evaluación del desempeño del trabajador es un punto muy delicado, ya que incide directamente sobre la moral de éste y sobre la eficiencia de la empresa; por lo tanto, debe considerarse cuidadosamente.

\section{Marco legal de la orga-} nización. La empresa es un conjunto de recursos organizados por el titular (emprendedor), con el fin de realizar actividades de producción o de intercambio de bienes o servicios que satisfacen las necesidades de un mercado en particular. Cuando se pretende constituir legalmente una empresa es importante tomar en cuenta, entre otros, los siguientes aspectos: número de socios, capital, responsabilidades, gastos de constitución, obligaciones fiscales, obligaciones laborales y políticas ecológicas. 
5. Finanzas. En la vida de cualquier empresa se debe saber cuál es su estado de salud es decir cómo se encuentra, las finanzas son las que representan los números de la empresa es decir la parte fuerte ya que mediante de ellas se evalúa a la empresa y se detectan fallas para su posterior corrección, es decir sabemos el estado de salud de la empresa, posibles enfermedades y curas (Rodríguez, 2011). La parte financiera tiene que incluir cuatro puntos principales, en los cuales se abordara todo lo que tiene que ver con la salud financiera de una empresa, dichos puntos son los siguientes:

\section{Sistema contable de la} empresa. Cuando se gestiona una nueva empresa existen puntos estratégicos en el diseño de dicha gestión es necesario planear el sistema contable que utilizara la empresa, el primer punto dentro del el sistema contable es seleccionar al responsable de dicho sistema, dicho responsable tendrá que estar capacitado para poder utilizar dicho sistema.

II. Costos y gastos. El costo es uno de los elementos más importantes para realizar las proyecciones o planeaciones de un negocio.
III. Estados financieros proyectados. Los estados financieros son resúmenes de resultados de las diferentes operaciones económicas de la empresa, en un periodo determinado o en una fecha específica futura.

\section{Indicadores financieros.} Los indicadores financieros son instrumentos que se utilizan para evaluar los resultados de las operaciones del negocio, toman como base los estados financieros proyectados. Los indicadores son muchos y muy variados, por lo que su aplicación y cálculo individual no es un elemento de toma de decisiones; es necesario aplicarlos simultáneamente y analizarlos para llegar a conclusiones válidas que faciliten el proceso administrativo de la empresa.

6. Medios de financiamiento. El emprendimiento en México está en desarrollo como parte de este desarrollo enfrenta una serie de problemas, el principal problema para los emprendedores es el financiamiento ya que, al no tener una garantía, pocas personas u instituciones confían en ellos en este subcapítulo se abordarán las posibles fuentes de financiamiento, con la finalidad de que el emprendedor coteje 
y elija la adecuada para su idea. Según Forbes México (Solís, 2018) existen tres métodos para poder financiar una idea y estos son los siguientes:

I. La triple F. Para muchos expertos en temas de emprendimiento la primera fuente de financiamiento para un emprendedor es la denominada triple $F$, por sus siglas en inglés (Friends, Family and Fools), esta opción de la triple $\mathrm{F}$ consiste en financiar la idea de negocio con la ayuda de amigos, familia o personas que crean en dicha idea. Al no contar con un respaldo financiero los emprendedores suelen utilizar esta opción.

\section{Financiamiento Guberna-} mental. Los fondos gubernamentales están disponibles para generar modelos de negocio y poder desarrollar proyectos, es decir, cuando está más avanzado que una simple idea. Asimismo, se usan para crear prototipos que ayuden a comercializar el producto o servicio en el mercado con la finalidad de generar un bien para la sociedad esto se puede materializar con nuevas fuentes de empleo ya sean directa o indirectamente. Algunos ejemplos de instituciones que cuentan con estos fondos son Financiera nacional y Banrural, INADEM, SAGARPA entre otras (Financiera, 2008).

III. Financiamiento bancario. Una opción real para un emprendedor es el financiamiento bancario (privado), este financiamiento se puede conseguir más rápido que los dos antes mencionados. Los bancos privados que se encuentran en el país cuentan con la opción de prestar dinero a personas físicas o morales a cambio de un porcentaje, estos porcentajes se cobran en base a una tasa anual, lo importante en este tipo de financiamiento es pagar a tiempo y ocupar dicho financiamiento con cautela, la banca comercial otorga financiamiento pero el acceso es limitado por lo que a la banca de desarrollo se le ha encomendado atender los proyectos prioritarios para el impulso, la recuperación y la modernización económica (Asociación de Bancos de México ABM, 2014).

\section{Metodología}

El trabajo realizado siguió una metodología de ocho fases, dichas fases son la elaboración de la 
naturaleza del proyecto, el mercado, producción, organización, finanzas, Financiamiento, puesta en marcha y la valoración de los resultados del funcionamiento de la microempresa. En la ilustración número uno se muestra la metodología ocupada para realizar la investigación.

\section{Figura 1. Metodología del trabajo.}

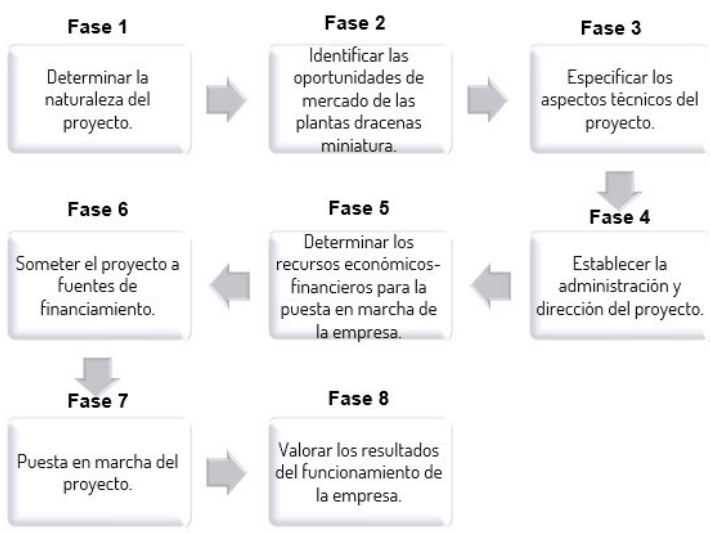

Fuente: Elaboración propia.

En la primera fase se realizó un estudio denominado naturaleza del proyecto en dicho estudio se realiza para definir la esencia de tu negocio, es decir, cuáles son los objetivos, cuál es la misión que persigue $y$ por qué se considera justificable su desarrollo. La naturaleza del proyecto es donde el emprendedor establece y define qué es su negocio. En esta fase se determinó ocho puntos importantes que la empresa debe de tener muy claro, estos puntos son: el producto que producirá, justificación, propuestas de valor, nombre, descripción (localización, tamaño), análisis FODA, visión, misión, objetivos, ventajas competitivas.

En la segunda fase se realizó una investigación en documentos de bibliotecas universitarias estás bibliotecas pertenecen al politécnico nacional de México, la universidad autónoma Chapingo y la universidad autónoma del estado de Morelos. La búsqueda también se realizó en revistas tales como Agro productividad, Horticultivos y bases de datos científicas como EBSCO HOST. En dichos lugares se encontraron trabajos donde mencionan lugares en los cuales se realizan actividades de comercialización de plantas ornamentales. Posteriormente se aplicó un estudio de mercado para conocer datos sobre los posibles clientes, el mercado destino y tener el conocimiento de la oferta y la demanda de las plantas dracenas miniaturas. 
En la tercera fase se realizó un estudio de producción el cual su objetivo fue determinar los aspectos técnicos que la microempresa requirió para su funcionamiento, para realizar dicho estudio se realizaron las siguientes actividades: especificar el producto a producir, describir el proceso de producción, establecer el diagrama de flujo de dicho proceso, determinar el equipo y las instalaciones, determinar la materia prima, capacidad instalada, diseño y distribución del vivero, mano de obra y establecer la mejora continua.

En la cuarta fase se realizó el estudio organizacional el cual su objetivo fue determinar los aspectos organizacionales que la microempresa utilizara, para obtener el estudio se realizaron las siguientes actividades, establecer la estructura organizacional, determinar las funciones de los puestos, realizar la captación de personal, determinar el desarrollo del personal, establecer los sueldos, la evaluación del desempeño y el marco legal de la organización.
En la quinta fase se realizó un estudio económico financiero el cual se realizó con el objetivo de conocer la viabilidad financiera de la microempresa, para realizar dicho estudio se determinaron los costos y gastos de la microempresa y se realizó la proyección de los estados financieros a cinco años para posteriormente evaluar el proyecto con indicadores financieros. En la fase número seis se realizó el sometimiento de la empresa a fuente de financiamiento, este proceso se realizó en las siguientes opciones: triple $f$, financiamiento gubernamental y financiamiento bancario. El sometimiento se realizó a través de entrevistas con diferentes interesados, convocatorias y visitas a instituciones bancarias.

En la fase siete se realizó la puesta en marcha del proyecto esta se realizó con la aplicación de un plan preoperativo y posterior a este se realizaron las actividades presupuestadas del proyecto en los estudios anteriores. La última fase de la metodología del proyecto consistió en realizar un análisis de lo implementado con lo presupuestado para con base esto saber el alcance del proyecto. 


\section{REsultados}

La investigación realizada arrojo una serie de resultados, estos surgieron por la aplicación de la metodología mencionada anteriormente, los resultados se dividen en ocho apartados los cuales se presentan a continuación. El primer resultado fue el conocimiento de la naturaleza del proyecto en el cual los puntos más sobresalientes fueron el nombre del proyecto (Verde Vida), el producto a producir (Dracena maicera miniatura), propuesta de valor (Plantas miniaturas, oferta de producto los 365 días, entrega de producto cada 15 días y producto libre de imperfecciones), se conocieron igual conceptos relacionados a la naturaleza del proyecto tales como la misión, visión, objetivos, ventajas competitivas y los aliados claves con los que contara el proyecto.

El segundo resultado fue el conocimiento de todos los aspectos relacionados al mercado del proyecto "Verde Vida", en este resultado los puntos más sobresalientes fueron, el conocimiento de 14 mercados ornamentales ubicados en los esta- dos de Puebla, Morelos y la Ciudad de México, la determinación del mercado ornamental de Atlixco de las Flores como el primer mercad destino para el proyecto "Verde Vida", se determinaron los objetivos y la segmentación del mercado, así como los potenciales competidores del proyecto. Posteriormente a estos resultados se obtuvo el estudio de mercado donde se aplicó un instrumento a 30 viveros del mercado ornamental destino, el resultado de la aplicación de dicho instrumento arrojo el conocimiento de una demanda insatisfecha de plantas dracenas miniatura, datos sobre los posibles clientes, distribución del mercado, precios y posibles cantidades requeridas por mes. Otros resultados relacionados con el mercado fueron la distribución del mercado del proyecto, plan de introducción al mercado, plan de ventas y datos relacionados con el producto (costo, precio, spot, etiqueta y envase). El tercer resultado fue el conocimiento de aspectos relacionados con la producción del proyecto "Verde Vida", en este resultado los puntos más sobresalientes fueron la determinación de los requerimientos de los productos, el proceso de producción, la tecno- 
logía a utilizar, equipo e instalaciones requeridas, ubicación del proyecto, distribución de planta, mano de obra y el plan preoperativo que se seguirá para poder poner en marcha el proyecto.

El cuarto resultado fue el conocimiento de aspectos relacionados con la organización del proyecto "Verde Vida", en este resultado los puntos más sobresalientes fueron el organigrama del proyecto, descripción de los puestos del proyecto, captación de personal (reclutamiento, selección, contratación, capacitación), sueldos de los puestos del proyecto, formal legal del proyecto y obligaciones legales del mismo. El quinto resultado fue el conocimiento de aspectos relacionados con las finanzas del proyecto "Verde Vida", en este resultado los puntos más sobresalientes fueron la inversión inicial del proyecto $(\$ 115,700.00)$, los estados financieros del proyecto, (presupuesto de ventas, presupuesto de producción, presupuesto de mano de obra, presupuesto de gasto indirectos de fabricación, presupuesto de gastos de operación, estado de costo de producción y ventas, estado de resulta- dos y el balance general). Se obtuvieron razones financieras para evaluar el proyecto, estas fórmulas fueron TIR (22\%) y VAN $(\$ 328,156.85)$.

El sexto resultado fue el conocimiento de aspectos relacionados con el financiamiento del proyecto "Verde Vida", en este resultado los puntos más sobresalientes fueron el sometimiento del proyecto, la primera opción de financiamiento fue la triple $F$, en esta opción se obtuvieron $\$ 45,000.00$ de dos personas (familiar y amigo), la segunda opción fue el financiamiento gubernamental donde se obtuvieron $\$ 3,000.00$ de un concurso (Eneit 2018 local), la última opción fue el financiamiento bancario, con esta opción se obtuvo un préstamo $(\$ 25,000.00)$ con un interés del $42.5 \%$ anual. En total se obtuvo un financiamiento de $\$ 73,000.00$ los cuales se utilizaron para realizar el plan preoperativo y la puesta en marcha del proyecto. El séptimo resultado fue el conocimiento de aspectos relacionados con la implementación del proyecto "Verde Vida", en este resultado los puntos más sobresalientes son el plan 
preoperativo, en el cual se invirtieron $\$ 17,240.00$, el segundo resultado fue la puesta en marcha del proyecto la cual se realizó en un lanzo de cuatro meses y nueve días, en dicha puesta en marcha se invirtió un total de $\$ 47,768.75$, se obtuvieron resultados de ventas en los meses de octubre y noviembre correspondientes a $\$ 7,000.00$, correspondientes a un periodo de cuarenta días.

El ultimo resultado fue la comparación de lo previsto con lo implementado donde se valoraron y compararon ambos resultados, las primeras diferencias fue el financiamiento conseguido $(\$ 115,700.00$ vs $\$ 73,000.00)$, el plan preoperativo, equipo $e$ instalaciones y salarios del personal contratado. La que sigue igual a lo presupuestado es lo correspondiente a la producción y ventas del proyecto. Con los resultados mencionados anteriormente se logró cumplir el objetivo de crear un proyecto capaz de producir y comercializar plantas dracenas miniatura para poder contribuir a la satisfacción de la demanda existente en el mercado ornamental.

\section{CONCLUSIONES}

El establecimiento de una microempresa ornamental soluciona dos problemas, el primero es la demanda insatisfecha de plantas dracenas miniatura en el mercado ornamental de Atlixco de las Flores, Puebla y el segundo es la falta de una empresa dedicada específicamente a la producción y comercialización de plantas dracenas miniatura en el municipio de Fortín de las Flores, Veracruz, México. El plan de negocios desarrollado representa una guía detalla para poner un nuevo negocio o mejorar una ya establecido en el caso del trabajo presentado denominado "Verde Vida", el plan de negocios, que se desarrollo fue la guía para poder concretar las ideas, encontrar un mercado destino, establecer los procesos adecuados para la producción del proyecto, determinar la organización y administración del proyecto, establecer los recursos financieros que se requieren para el funcionamiento del proyecto, esto para poder someter el proyecto a financiamiento. 
Con la finalización de este trabajo se generaron dos fuentes de empleo para los habitantes de dicho municipio hablando internamente $y$ externamente con la compra de la materia prima a productores del municipio. Con el funcionamiento de la microempresa se espera poder marcar una pauta entre los productores ornamentales del municipio de Fortín de las Flores, el modelo establecido en el trabajo se puede mudar a los negocios de los productores ornamentales de este municipio, esto se debe de realizar de la mano con los gobiernos locales e instituciones que apoyen al campo, si se aplica correctamente el modelo, esto representara un detonante en la generación de empleos y una derrama económica, lo cual contribuirá al desarrollo de la zona ornamental ubicada en la zona centro del estado de Veracruz.

\section{REFERENCIAS}

AIPH. (24 de Julio de 2014).

AIPH.ORG. Obtenido de

http://aiph.org/aiph_new/aiph-andunionfleurs-launch-internationalstatistics-flowers-andplants-2014/

Aldana, V. M. (2010).

Competitividad de la pequeña empresa del sector ornamental en Atlixco, Puebla. Red de Revistas Científicas de América Latina y el Caribe, España y Portugal, 26-35.

Asociación de Bancos de México ABM, A. (2014). Obtenido de https://abm.org.mx/la-banca-enmexico/

Balanko-Dickson, G. (2008). Cómo preparar un plan de negocios exitoso. Mexico: McGraw-Hill Interamericana.

Bouchet E.R., F. C. (2007). Efecto de las dimensiones de un invernadero sobre la temperatura interna en periodos cálidos. Revista Científica Agropecuaria 11 (2)., 111-119.

Chalate-Molina, H. (Marzo de 2008). Programa estrategicó de necesidades de investigación y trasferencia y trasferencia de tegnología de la cadena productiva horticultura ornamental en el estado de Veracruz. Veracruz. 
Filion, L. J., Cisneros, L. F., \& Mejía-Morelos, J. H. (2011).

Administración de PYMES. México.: PEARSON EDUCACIÓN.

Financiera, N. (2008). NAFIN. Obtenido de http://www.nafin.com.mx/portalnf/co ntent/productos-yservicios/programasempresariales/programa-creditopyme.html

Horticultivos, R. (15 de Diciembre de 2011). La industria de las ornamentales en México. Obtenido de

http://www.horticultivos.com/788/laindustria-de-las-ornamentales-enmexico/

Infoagro Systems, S. (2012).

Infoagro. Obtenido de http://www.infoagro.com/flores/plant as_ornamentales/dracena.htm

Rivera, F. (13 de Marzo de 2015).

Milenio. Obtenido de http://www.milenio.com/df/flores_14 febrero-

demanda_flores_14_febreroproduccion_nacional_flores_0_4637 53861.html

Rodríguez, R. E. (2011). EL EMPRENDEDOR DE ÉXITO (Vol. Cuarta edicción.). Mexico D.F.: McGraw-Hill.

Secretaría de Agricultura, G. D. (2014). SAGARPA. Obtenido de https://www.gob.mx/sagarpa/prensa/ participan-agroempresarios-en-laexpo-paisajismo-y-vida-sustentable2014
Solís, A. (Marzó de 22 de 2018).

Forbes México. Obtenido de https://www.forbes.com.mx/estasson-las-principales-fuentes-definanciamiento-paraemprendedores/

Tecnoagro. (2010 de Julio de 21). Tecno Agro. Obtenido de https://tecnoagro.com.mx/revista/20 10/no-61/produccion-de-flores-decorte-y-plantas-de-ornato-enmaceta/

Xochimilco, D. (Noviembre de 2008). Tu voz. "Órgano de Difusión de la Delegación Xochimilco". CDMX, Mexico.

Yanai., P. J. (2012). Centre., Cut Flowers and Ornamental Plants. International Trade. Issue 33. 